\title{
Non-Contact Monitoring of Heart and Lung Activity by Magnetic Induction Measurement
}

\author{
M. Steffen, S. Leonhardt
}

In many clinical applications, the monitoring of heart and lung activity is of vital importance. State-of-the-art monitoring involves the use of electrodes or other contact based sensors (electrocardiogram (ECG), impedance cardiography (ICG), pulse oximetry or equivalent). With the equipment that is used, side effects like skin irritation, difficult application or additional cabling may occur. In contrast, this paper describes a method for non-contact monitoring of heart and lung activity, which is solely based on magnetic induction. This method allows simultaneous monitoring of heart and lung activity, and has the potential of an integrated application in a personal healthcare scenario. To illustrate the performance, a simple test-setup has been developed and the first results are presented here (some of which have been previously presented on the Poster 2008 [10]).

Keywords: Magnetic induction, non-contact, monitoring, heart, lung.

\section{Introduction}

Many first and second world countries are facing dramatic demographic changes. Mainly due to longer life expectancy and reduced natality, societies are growing older [1]. As ageing is accompanied by increasing costs for medical care, there are increasing efforts to develop smart integrated medical devices to support people with health problems in their everyday life, and especially in their domestic environment. Within the personal healthcare scenario, monitoring of heart and lung activity is of special interest. Continuous 24/7 monitoring techniques for an intelligent warning system, making use
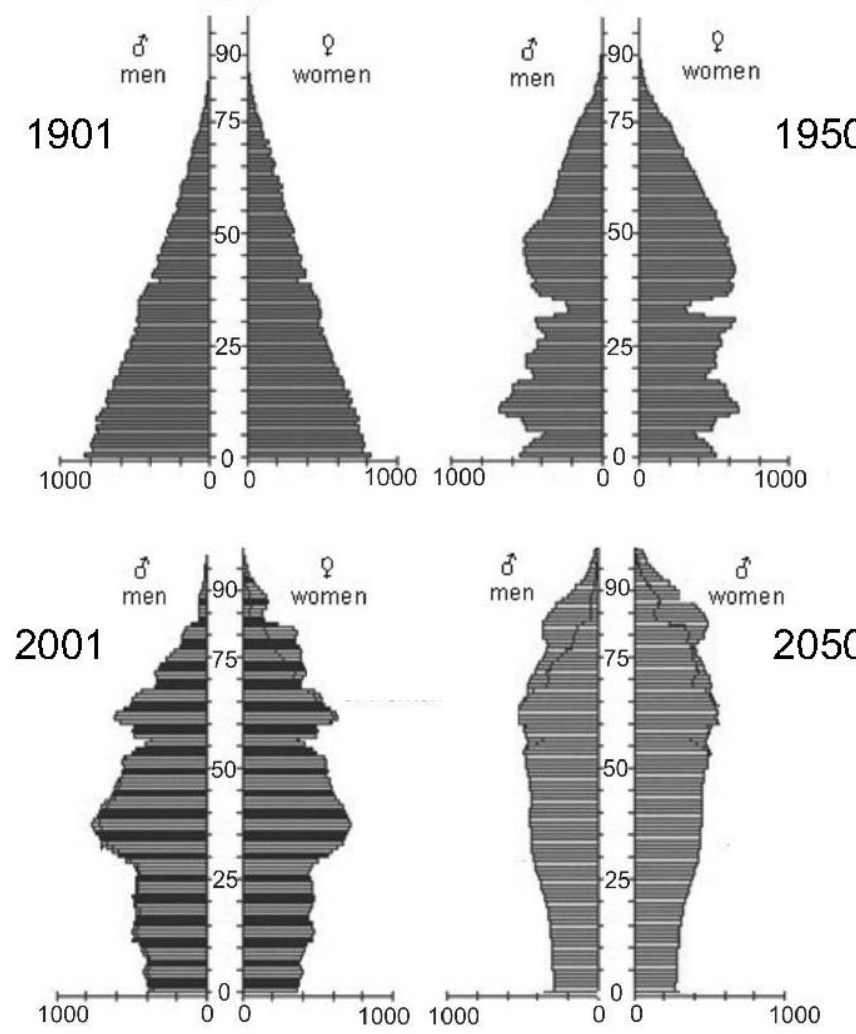

Fig. 1: Shapes of the German population pyramid changing in time (modified from [1]) of a range of sensor information are required for patients at risk (e.g. of post myocardial infarction).

Special focus needs to be given to the time when patients are alone at home, and especially when they are sleeping. During this period, which covers roughly $1 / 3$ of the day, neither the patient nor his relatives or neighbours can raise an alarm. The need for long-term monitoring is accompanied by further constraints: for example, movement should not be restrained, and long-term skin irritation must be avoided. At best, the measurements should not be noticeable.

From a physical point of view, potentional sensors for imperceptible mobile monitoring can be based on capacitive, magnetic or optical measurement techniques. An approach based on magnetic induction is presented here.

\section{Background}

A healthy life is based on the fact that the heart pumps blood through the body, and breathing exchanges the air inside the lungs while changing the lung volume. The effect of these two mechanical actions is to a shift well-conducting matter (blood) and poorly-conducting matter (air) inside the chest region. As shown by Tarjan [2], a measurement system based on magnetic induction can monitor these changing impedances in the human body. From Maxwell's Theory [3], it is principally known that:

$$
\begin{aligned}
& \nabla \times \vec{E}=\frac{\partial}{\partial t} \vec{B} \\
& \vec{J}=\kappa \cdot \vec{E} \\
& \nabla \times \vec{H}=\vec{J}+\frac{\partial}{\partial t}(\varepsilon \cdot \vec{E})
\end{aligned}
$$

where $\nabla$ is the Nabla-operator, $E$ denotes the electric field, $B$ denotes magnetic induction and $J$ represents current density. As the re-induced field is related to the impedance distribution inside the body, the impedance changes directly affect the re-induced voltage in the whole area (see Fig. 2).

If the frequency and permittivity $\varepsilon$ is low, the displacement current can be neglected. Previous works have already indicated that this approach is promising for heart activity [4] and lung activity [5] monitoring. However, due to the superposi- 


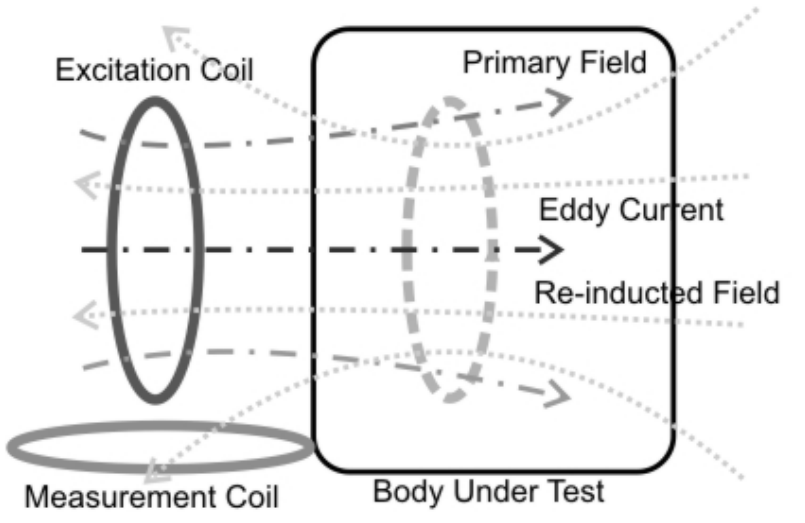

Fig. 2: Concept of magnetic induction measurements [9]. Excitation field penetrates the body. Eddy current is induced and the resulting re-induced field is measured.

tion of the re-induced fields, simultaneous measurement of heart and lung activity is rather difficult. As a simple coil setup might not be sufficient in all cases (see [7]), a multi-coil setup has been developed and tested.

\section{Measurement setup}

The structure of the resulting measurement setup is depicted in Fig. 3, and consists of several blocks:

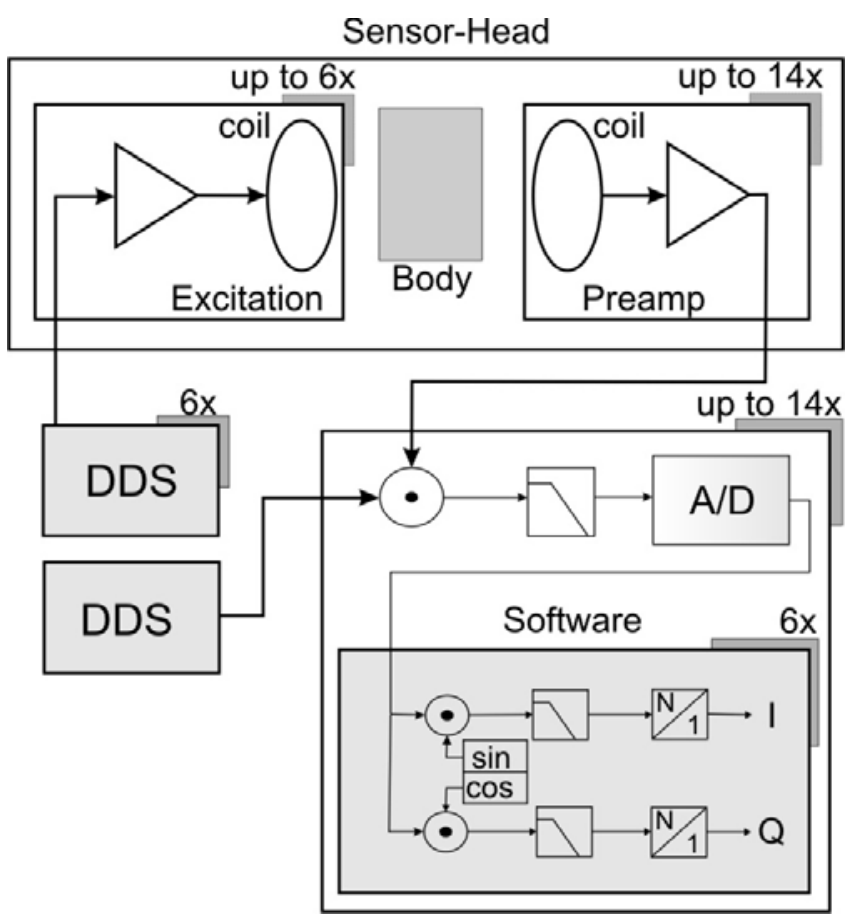

Fig. 3: Overview of the measurement hardware. Excitation frequencies are generated by DDS synthesizers and feed to the excitation-coils. The resulting field penetrates the body and produces the re-induced field. This is picked up by the measurement-coils, pre-amplified and demodulated in a combined hardware/software approach (see [9]).

1. A signal generator (Direct Digital Synthesis - DDS) to provide all required frequencies
2. Multiple parallel receivers in which the HF signals are down-converted into the baseband.

3. A measurement PC, which performs $\mathrm{A} / \mathrm{D}$ conversion and all further signal-processing steps.

4. A sensor head, which holds the actual excitation and measurement coils.

5. A control and timing block, which generates all required reference-clocks and controls the signal generator and receivers.

6. Some reference sensors, including a pulse plethysmograph (PPG), an airflow sensor and a DCF Radio-Clock-Module.

A sinusoidal signal from the signal generator is amplified and feed to the excitation coils and thus generating the excitation field. This field penetrates the body and induces an eddy voltage. This eddy voltage drives an eddy current, which is influenced by the excitation field as well as the tissue impedance. This eddy current leads to a re-induced magnetic field, which superimposes the excitation field. The resulting field is measured by the receiver coils.

The voltage induced by the magnetic field into the receiver coils is pre-amplified and then fed to the amplifier and demodulator stage. Here it is mixed down to a $20-100 \mathrm{kHz}$ baseband signal. This signal is sampled and $\mathrm{A} / \mathrm{D}$ converted. The further processing is carried out in the software on the measurement PC.

The hardware described above was used in combination with the coil array, which is composed of two excitation coils (lying) and three measurement coils (standing) and this provides six independent channels of inductive coupling.

Experimentally, the sensor head was placed beneath a sun lounger, on which a healthy adult volunteer was resting, as shown in Fig. 4.

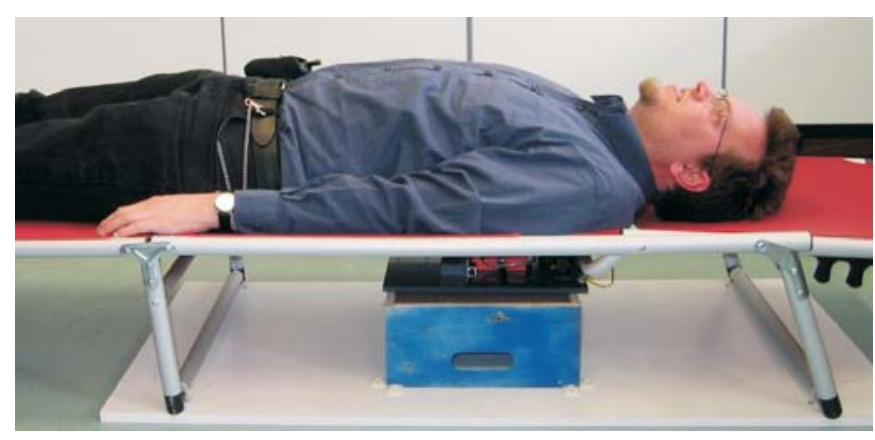

Fig. 4: Picture of the measurement setup

\section{Results}

Using this setup, the magnetic induction of the previously mentioned six channels was simultaneously recorded, together with the signals of a pulse plethysmograph (Nellcore $@$ DS-100a fingerclip \& self-made electronic) and an airflow sensor based on differential pressure measurements (General Electric $@$ Flow resistance \& Hoffrichter CPS 1B differential pressure sensor).

For illustration, Fig. 5 shows all recorded signals during a selected period of timed. 


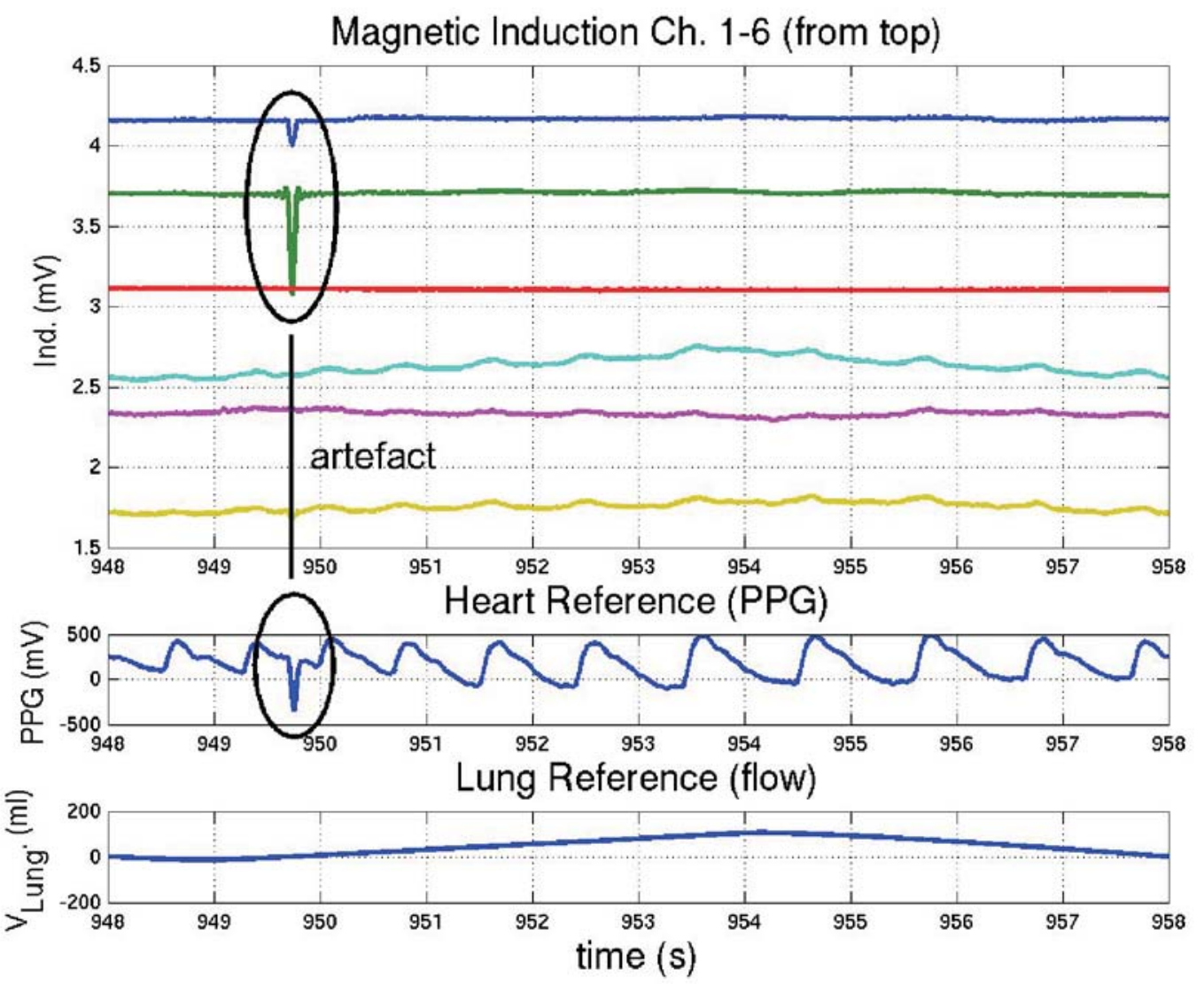

Fig. 5: Overview on all signals measured simultaneously (from top: Induction Channels 1-6 (mV), PPG, Airflow (ml)). At $950 \mathrm{~s}$ the inductive signal and the heart reference show an artefact presumably due to body motion.

In this dataset it is especially interesting that the artefact shortly before $t=950 \mathrm{~s}$ seems to affect both the PPG and the magnetic induction. Especially the strong appearance in channel 1 which otherwise shows little heart related content
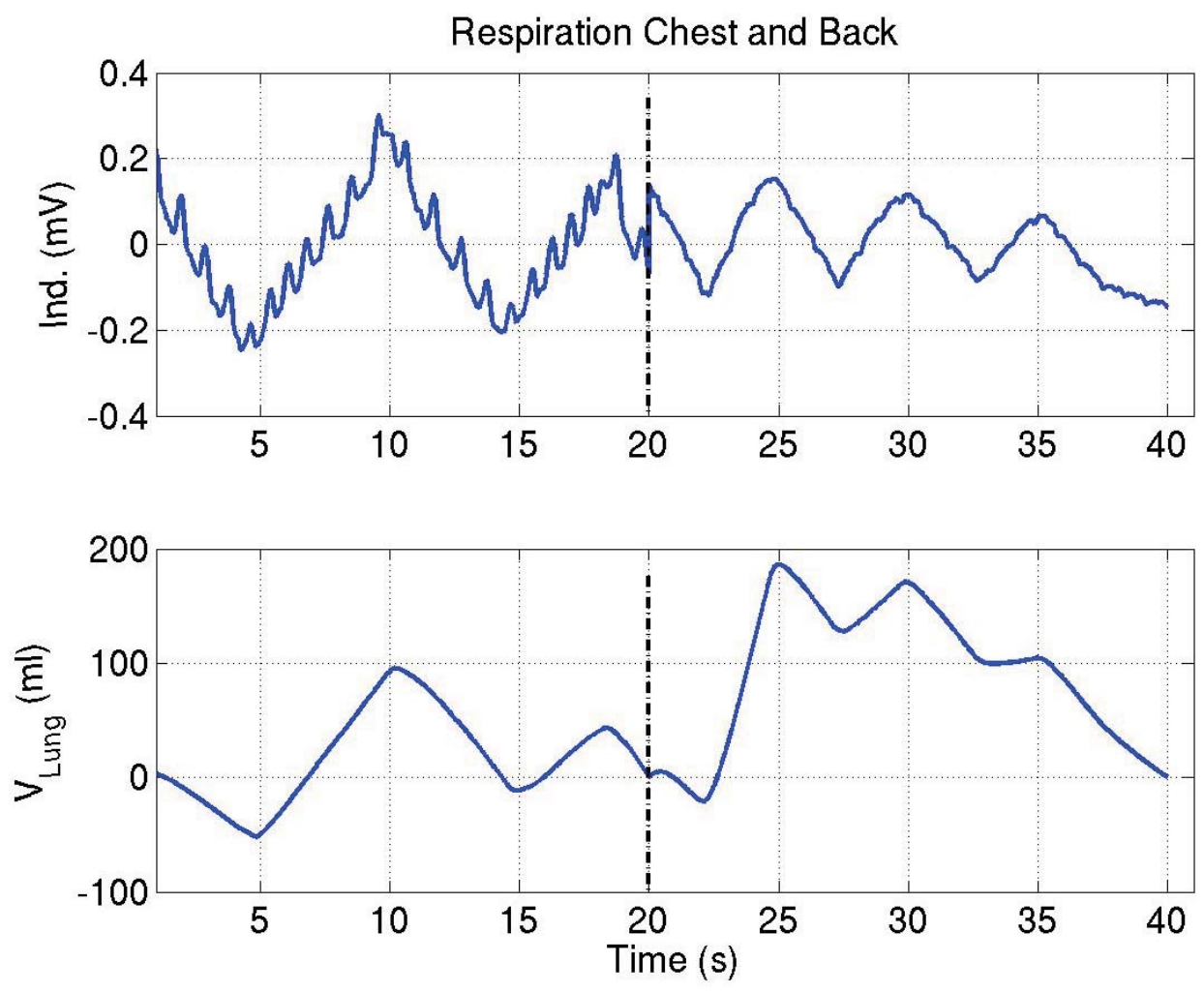

Fig. 6: Overview on the respiration related signals measured from chest and back 
makes it rather likely that this is a movement artefact and not an extra-systole.

To find out whether monitoring in a bed is feasible in principle, measurements were undertaken with four different body positions:
1. Lying on the chest
2. Lying on the left side
3. Lying on the back
4. Lying on the right side

In order to keep this preliminary testing as simple as possible, no further disturbance, e.g., by motion artefacts, was introduced, and moving metal parts were removed from the measurement area. During the measurement no mobile phones were carried though no negative effects on the measurement due to mobile phones have ever been experienced. Magnetic shielding of the measurement setup is not required.

In all four positions, respiratory frequency monitoring was possible without any problem, as shown in Fig. 6 and Fig. 7
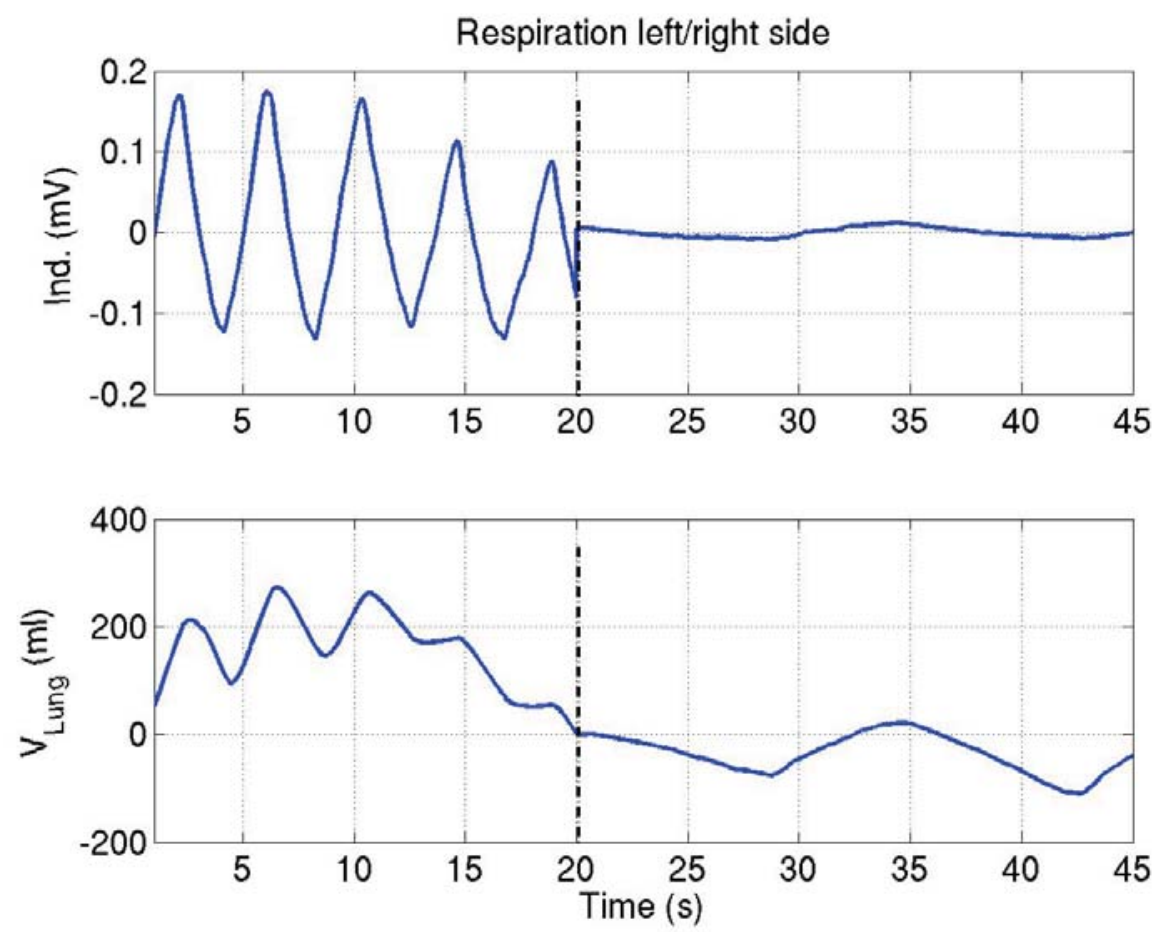

Fig. 7: Overview on the respiration related signals measured from left and right side
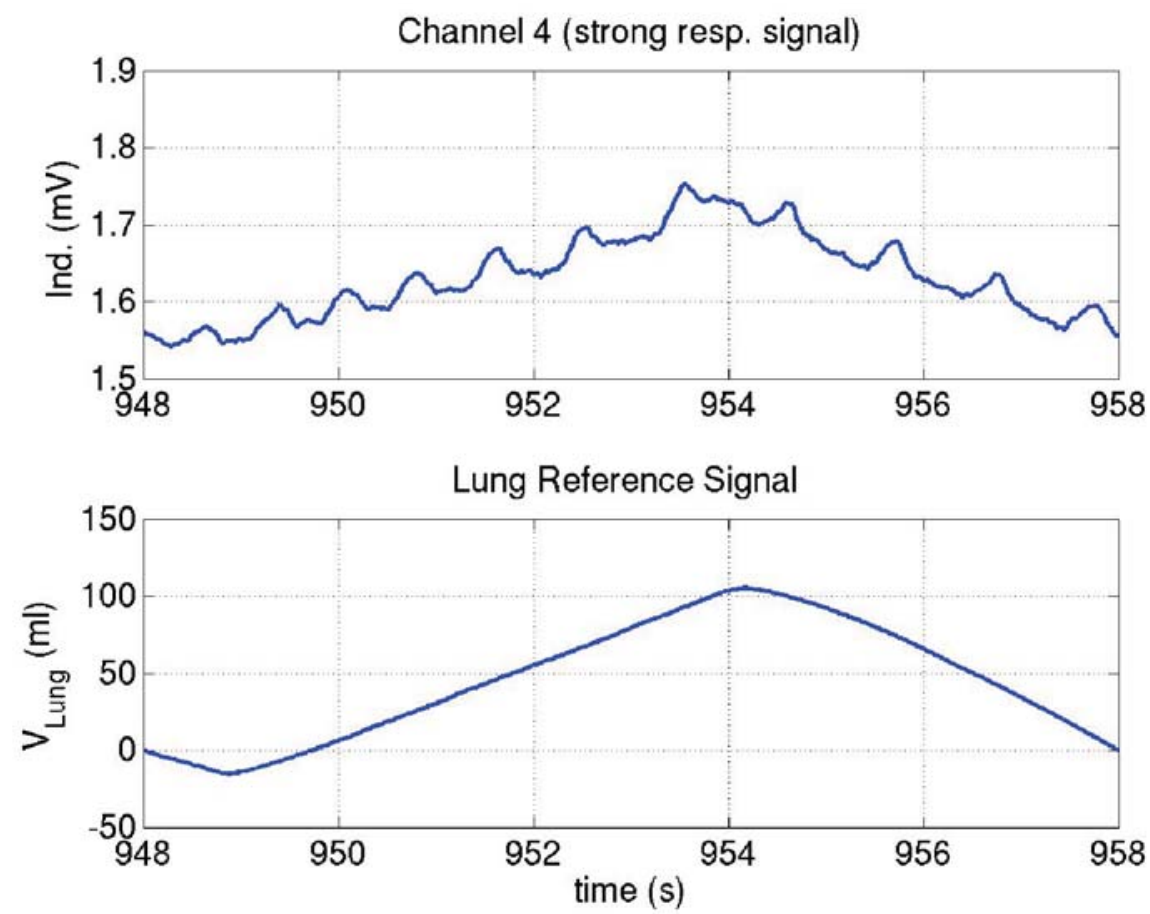

Fig. 8: Comparison inductive measurement (Ch. 4) vs. respiratory reference (Volunteer lying on the chest) 
which shows the four positions for $20 \mathrm{~s}$ each. From the experience in [8], it is likely that the difference between the induction signal and the reference signal is not due to errors in the inductive monitoring, but rather due to drift, saturation and leakage in the reference sensor.

As expected heart-activity monitoring is more demanding, though in position 1 (chest) band pass filtering with cut-off-frequencies of $0.75 \mathrm{~Hz}$ and $5 \mathrm{~Hz}$ were sufficient to derive the signals shown in Fig. 8 and Fig. 9, where pulse plethysmograph and the magnetic induction bear strong similarity.

Similar results where obtained when the volunteer was lying on the left side, as the distance to the centre of the heart is slightly larger the resulting inductive signal is a bit weaker, as shown in Fig. 10.

For the other positions further processing was required. This especially utilizes the availability of multiple simultaneously recorded induction channels.

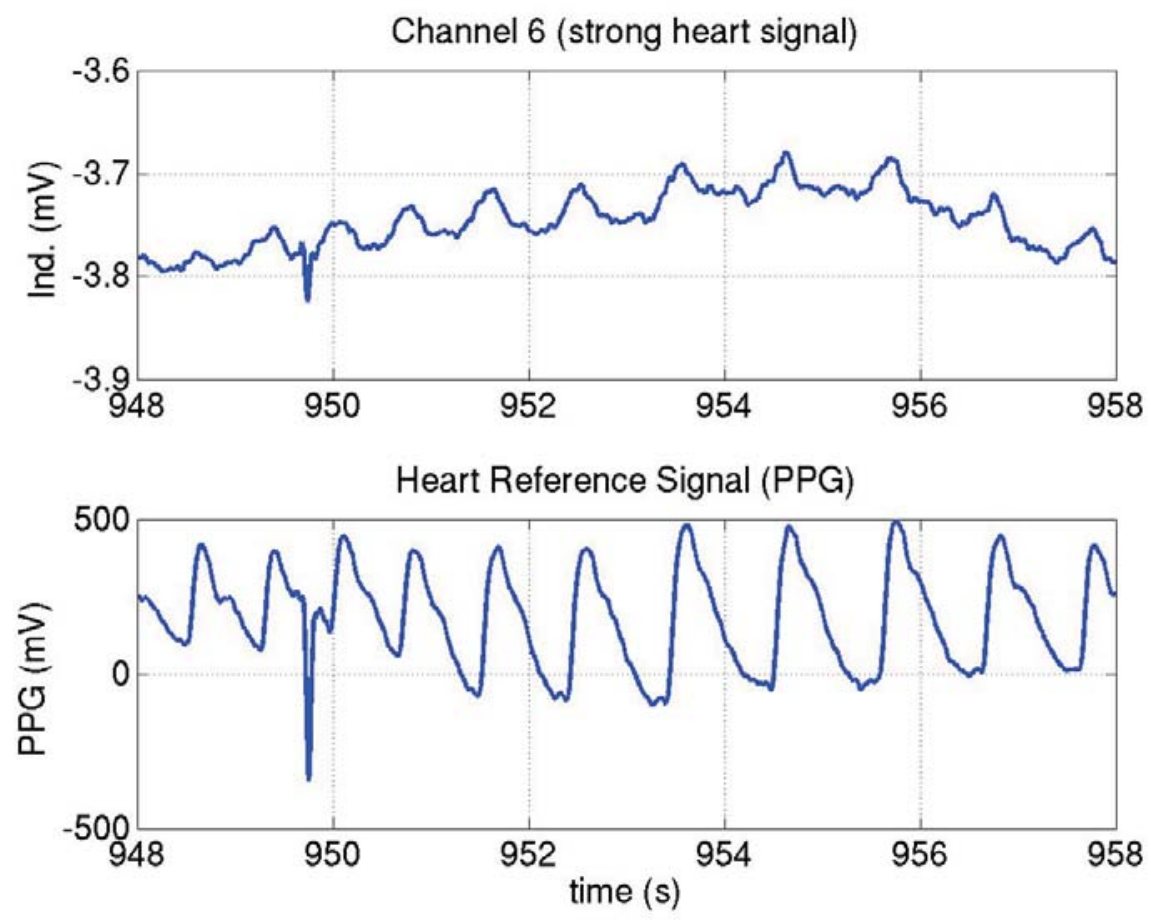

Fig. 9: Comparison inductive meas. (Ch. 4) vs. heart reference (PPG) (Volunteer lying on the chest)
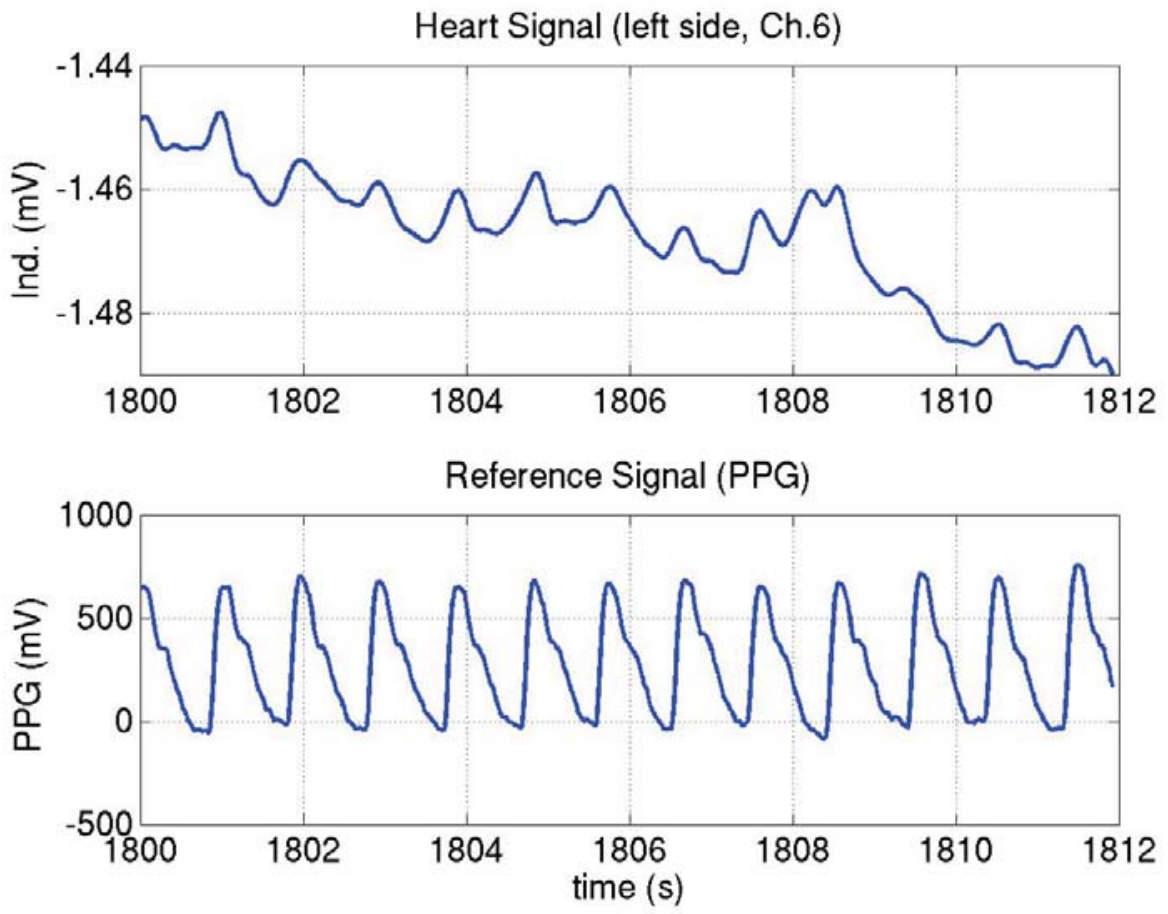

Fig. 10: Comparison inductive meas. (Ch 6) vs. heart reference (PPG) (Volunteer on it left side ) 
In the case of the volunteer lying on his back, noise was reduced by subtracting channel 3 from channel 4 . The resulting signal and it comparison to the reference signal are shown in Fig. 11 and Fig. 12.

In the case of the volunteer lying on the right side, signal noise was reduced by combining two channels (Ch. 4 and
Ch. 6), which lead to the signals depicted in Fig. 13 and Fig. 14.

In all cases, the required scaling of the channels considered was performed manually. Automated channel selection and combination is under development.
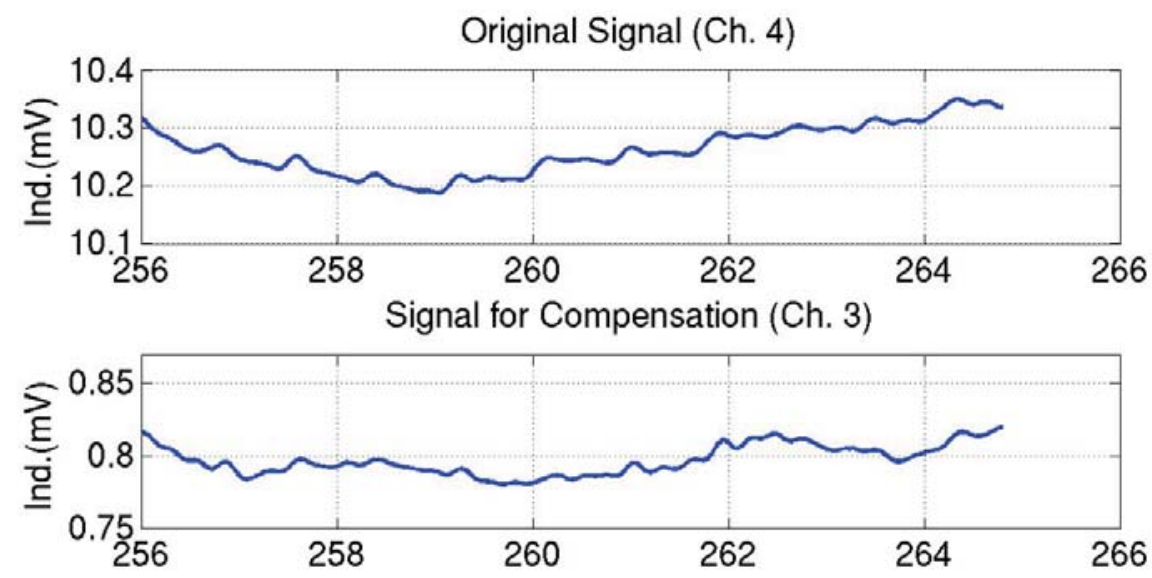

Ch. 4 - Ch. 3

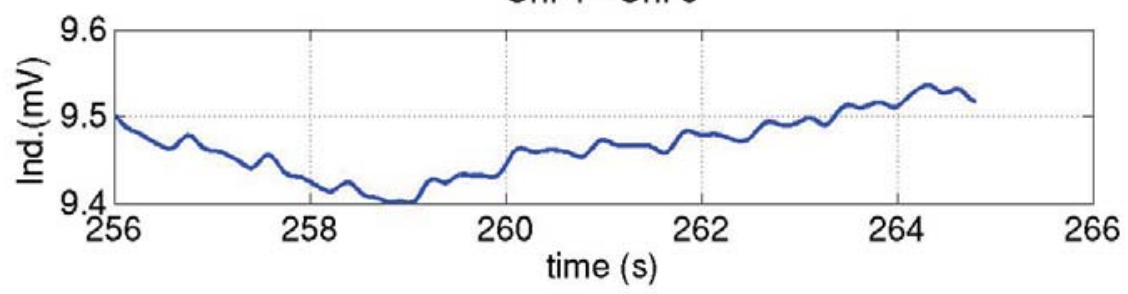

Fig. 11: Drift reduction / compensation in (Ch. 4 h. 3), result shown below (Volunteer in back position)

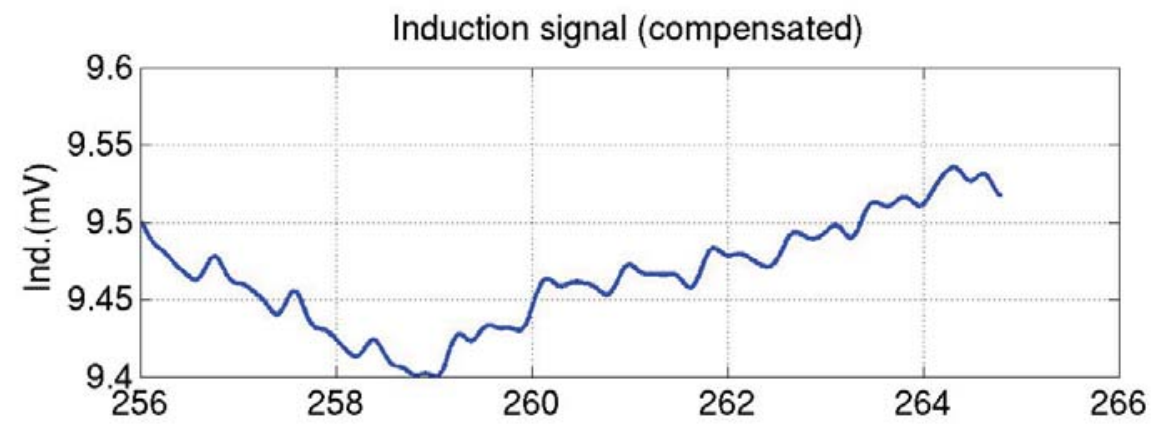

Reference Signal (PPG)

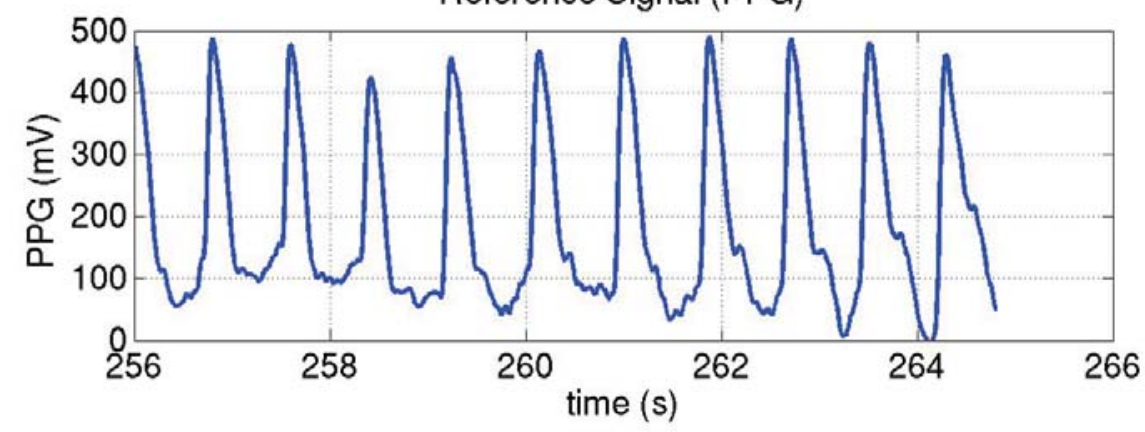

Fig. 12: Comparison inductive meas. (drift compensation Ch4. - Ch. 3) vs. heart reference (PPG) (Volunteer in back position) 
Ch. 4

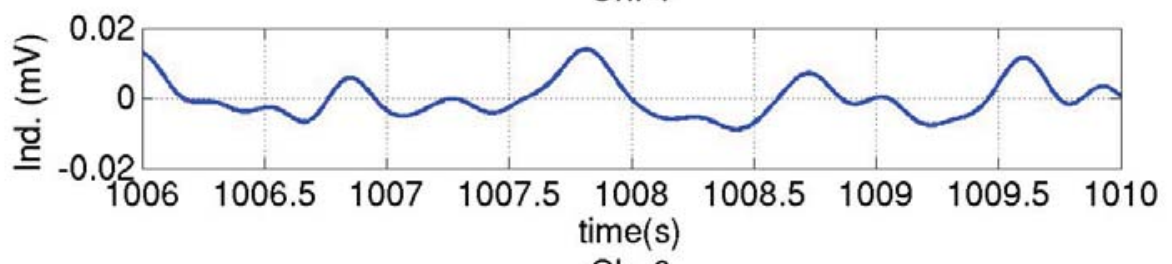

Ch. 6

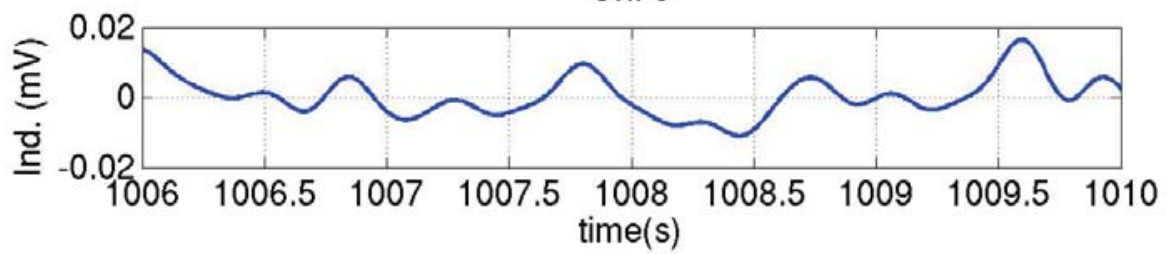

Ch. $4+$ Ch. 6

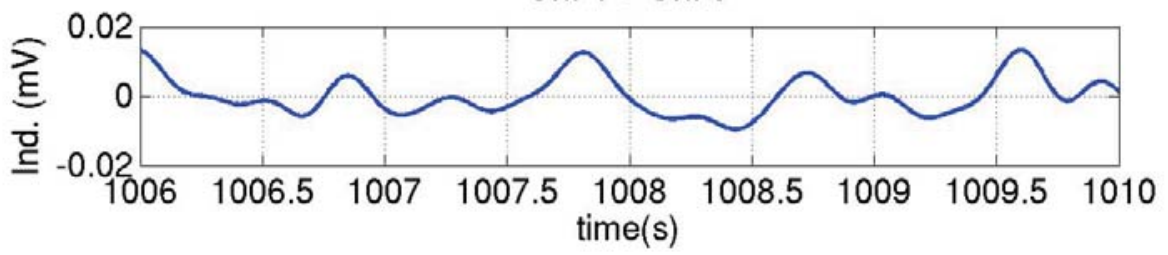

Fig. 13: Noise reduction, combining Ch. 4 and Ch. 6, result shown below (Volunteer lying on the right side)
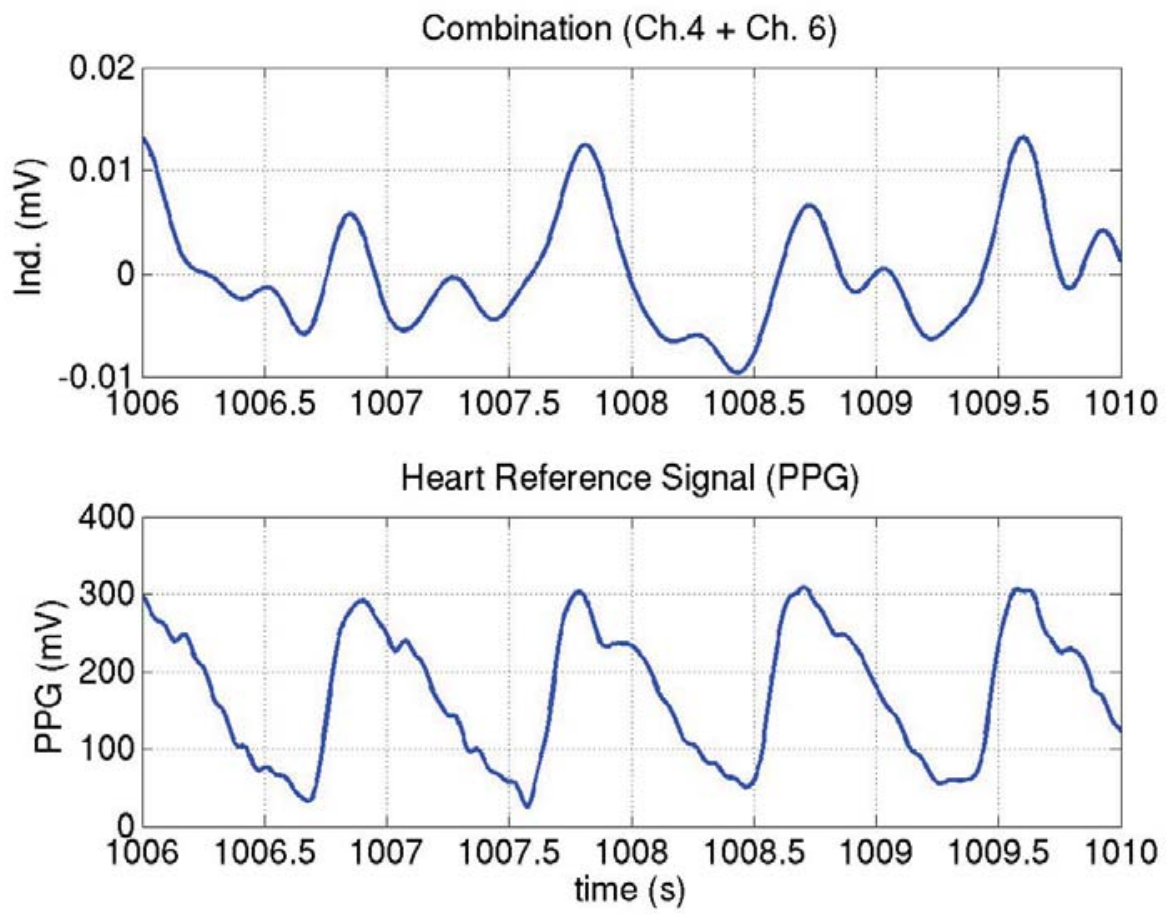

Fig. 14: Comparison inductive meas. (Combination Ch. $4+$ Ch. 6) vs. heart reference (PPG) (Volunteer lying on the right side)

\section{Conclusion}

The elimination of electric contact between a medical device and the patient's body is a promising idea, and this paper presents one feasible approach to non-contact monitoring, based on magnetic induction. This approach is especially suitable for integration into a normal bed.
Though the measurements still required some manual post-processing, lung and heart related signal contents were detectable and distinguishable in all positions.

Further work will be done on automatic scaling and multivariate combination of the measured

channels and on taking into account motion artefacts and EM 
interference. The problem of respiratory and heart rate detection will also be addressed.

\section{Acknowledgments}

This work was supported in part by the Aachen Competence Centre for Biomedical Engineering (AKM) and the Federal Ministry for Education and Research (BMBF), Germany. Ongoing research is supported by the German Research Foundation (DFG, STE 1811/1-1).

\section{References}

[1] Statistisches Bundesamt (Federal German Office for Statistics), Bevölkerung Deutschlands bis 2050 ("German population til 2050"). www.destatis.de, 2003.

[2] Tarjan, P., McFee, R.: Electrodeless Measurements of the Effective Resistivity of the Human Torso and Head by Magnetic Induction, IEEE trans. on Biomedical Engineering, 1968.

[3] Maxwell, JC.: Lehrbuch der Elektrizität und des Magnetismus, 1. Ed., Berlin: Springer, 1883.

[4] Guardo, R., Trudelle, S., Adler, A., et al.: Contactless Recordings of Cardiac Related Thoracic Conductivity Changes. Proceedings of the Engineering in Medicine and Biology Conference, 1995, Canada, p. 1581-582.

[5] Richter, A., Adler, A.: Eddy Current Based Flexible Sensor for Contactless Measurement of Breathing. Proceedings IMTC 2005 Instrumentation and Measurement, Ottawa, Canada, 2005.
[7] Steffen, M., Leonhardt, S.: Requirement Estimation of Simultaneous Heat and Lung Activity Monitoring with Magnetic Impedance Tomography Systems. WC on Med. Phy. and Biomed. Eng. 2006, Korea.

[7] Steffen, M., Aleksandrowicz, A., Leonhardt, S.: Mobile Non-Contact Monitoring of Heart and Lung Activity, IEEE Trans. Biomed. Circ. and Sys., Vol. 1 (2007), No. 4.

[8] Steffen, M., Heimann, K., Bernstein, N., Gonzalo, D., Leonhardt, S.: Kontaktloses Monitoring von Vitalparametern bei Neugeborenen. 141. Jahrestagung der Deutschen Gesellschaft für Biomedizinische Technik im VDEBMT 2007, 2007.

[9] Steffen, M., Heimann, K., Bernstein, N., Leonhardt, S.: Multichannel Simultaneous Magnetic Induction Measurement System (MUSIMITOS). Phys. Meas. Vol. 29 (2008), No. 6, p. 291-306.

[10] Steffen, M.: Non-Contact Monitoring of Heart and Lung Activity by Magnetic Induction Measurement, Student Conference Poster 2008, Prague 2008.

\footnotetext{
Matthias Steffen

e-mail: steffen@hia.rwth-aachen.de

Steffen Leonhardt

Chair for Medical Information Technology

RWTH Aachen University

Aachen Pauwelsstr. 20, Germany
} 